\section{Multipotential differentiation ability of GATA-1-null erythroid-committed cells}

\author{
Kenji Kitajima, Jie Zheng, Hilo Yen, \\ Daijiro Sugiyama, and Toru Nakano ${ }^{1}$ \\ Department of Pathology, Medical School and Graduate \\ School of Frontier Biosciences, Osaka University, Suita, \\ Osaka 565-0871, Japan
}

GATA-1, a zinc finger transcription factor, has been believed to be indispensable for the survival of proerythroblasts. However, we found that GATA-1-null proerythroblasts could survive and proliferate on OP9 stroma cells in the presence of erythropoietin. Furthermore, myeloid and mast cells were induced from the GATA-1-null proerythroblasts by the stimulation of granulocyte-macrophage colony-stimulating factor (GM-CSF) and interleukin-3 (IL-3), respectively, but lymphoid differentiation was not achieved by in vivo transfer. Thus, without activity of the transcription factor required for terminal differentiation, even relatively mature and committed cells proliferate continuously with the differentiation capacity to other lineages. Our data suggest that GATA-1 is a critical transcription factor to fix erythroid progenitors to the erythroid lineage.

Received December 10, 2005; revised version accepted January 1, 2006.

Hematopoiesis is a cell differentiation process tightly controlled by various lineage-specific transcription factors (Cantor and Orkin 2001). Among them, GATA zinc finger transcription factors are one of the most well-studied transcription factors, and three members of the family, GATA-1, GATA-2, and GATA-3, are involved in hematopoiesis (Cantor and Orkin 2001). GATA-1, the founding member of the GATA family, participates in the expression of numerous genes involved in erythropoiesis (Morceau et al. 2004; Welch et al. 2004; Ferreira et al. 2005). Gene targeting analysis has revealed that GATA-1 is an essential transcription factor for erythropoiesis (Pevny et al. 1991, 1995; Weiss et al. 1994; Fujiwara et al. 1996). GATA-1-null embryonic stem (ES) cells gave rise to definitive erythroid precursors. But the GATA-1-null erythroid cells were arrested at the proerythroblast stage in vitro and failed to contribute to adult red blood cells in chimeric mice. Therefore, GATA-1-null mice showed complete ablation of erythropoiesis owing to the arrested maturation and apoptosis of erythroid precursors at the proerythroblast stage. Meanwhile, overexpression of GATA-1 reprograms my-

[Keywords: Cell differentiation; transcription factor; GATA-1; erythroid cell]

${ }^{1}$ Corresponding author.

E-MAIL tnakano@patho.med.osaka-u.ac.jp; FAX 81-6-6879-3729. Article and publication are at http://www.genesdev.org/cgi/doi/10.1101/ gad.1378206. eloid cells to undergo erythroid and megakaryocytic differentiation (Kulessa et al. 1995; Iwasaki et al. 2003).

In vitro differentiation of ES cells to blood cells is a powerful tool for the analysis of hematopoietic differentiation and development because it enables us to analyze the direct consequences of the effects of gene disruption. There are basically two methods used for in vitro differentiation: the conventional embryoid body formation method and the coculture of ES cells on a stroma cell line, such as OP9. The coculture of ES cells on OP9 cells, the OP9 system, has several advantages, including the quantitative analysis of terminally differentiated blood cells by providing an appropriate hematopoieitc microenvironment (Nakano et al. 1994, 1996; Kitajima et al. 2002; Tanaka et al. 2004; Zheng et al. 2006). In the OP9 system, various types of blood cells, including erythrocytes, are developed, and the time course of the development precisely recapitulates normal erythroid development (Nakano et al. 1996; Kimura et al. 2000).

In this study, we examined the erythroid differentiation from GATA-1-null ES cells using the OP9 system. Unexpectedly, GATA-1-null erythroid cells similar to proerythroblasts continued to proliferate on OP9 cells in the presence of erythropoietin (EPO). A small percentage of these presumably erythroid-committed cells came to express myeloid markers spontaneously. Furthermore, the cells differentiated to mature myeloid cells and mast cells when EPO was replaced by granulocyte-macrophage colony-stimulating factor (GM-CSF) or interleukin-3 (IL-3), respectively. On the other hand, lymphoid differentiation was not detectable after in vivo transfer of the cells. In other words, the GATA-1-null proerythroblasts retained the differentiation ability to myeloid but not to lymphoid lineages. These data show that "commitment" takes place but is not feasible without the transcription factor essential for terminal differentiation.

\section{Results and Discussion}

\section{Proliferation of erythroid cells derived from GATA-1-null ES cells}

Differentiation to erythroid cells from wild-type and GATA-1-null ES cells was carried out using the OP9 system in the presence of EPO (Nakano et al. 1996; Kimura et al. 2000). Wild-type ES cells differentiated to mature erythrocytes and disappeared by completing terminal differentiation $\sim 2$ wk after the differentiation initiation. In contrast, GATA-1-null ES cells gave rise to cells that continued to proliferate for $>3$ mo in a logarithmic manner (Fig. 1A). Morphologically, the cells were similar to proerythroblasts in normal erythropoiesis (Fig. 1B). The GATA-1-null cells could not survive on OP9 cells without EPO or in the presence of EPO without OP9 cells, showing that the cells were strictly dependent on both EPO and OP9 stroma cells (data not shown). A significant proportion $(15 \%-40 \%)$ of the GATA-1-null cells continuously died due to apoptosis in our culture (Fig. 1C). It has been reported that under the GATA-1-null condition, erythroid cells die at the proerythroblast stage by apoptosis (Weiss and Orkin 1995; Pan et al. 2005). Apoptosis of the GATA-1-null cells under our culture 
A

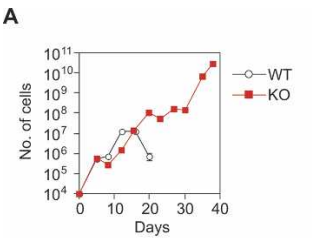

D

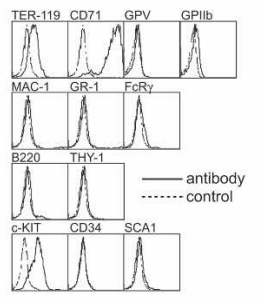

$\mathbf{F}$

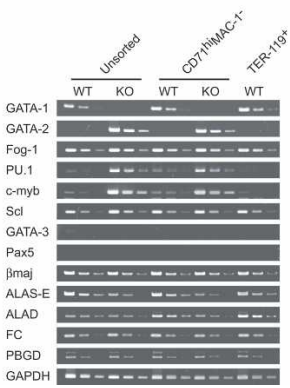

E

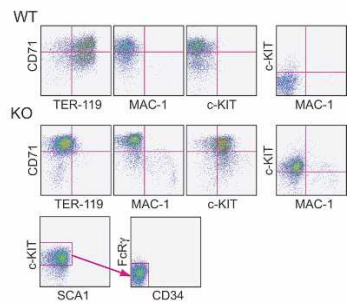

B

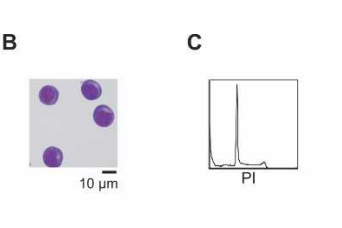

\section{(1)}

Differentiation of GATA-1

morphology, the vast majority of GATA-1-null cells possess erythroid characteristics and presumably belong to the stage of proerythroblasts.

Next, expression of various hematopoietic transcription factors between wild-type and GATA-1-null cells in the unsorted and sorted CD $71^{\text {high }} \mathrm{Mac}-1^{-}$fractions were analyzed (Fig. 1F). For comparison, wild-type TER-119 ${ }^{+}$ erythroid cells at day 12 after coculturing on OP9 cells in the presence of EPO were used, because day 12-induced cells were morphologically similar to the GATA-1-null erythroid cells. GATA-2 expression level of the GATA1 -null proerythroblasts was fivefold more than that of day 12 erythroid cells. Expression of the transcription factors Pax5 and GATA-3 involved in B- and T-cell differentiation was not observed. However, PU.1, a crucial regulator for macrophage, mast cells, and B cells (Scott et al. 1994; DeKoter and Singh 2000; Walsh et al. 2002), was highly expressed in the GATA-1-null proerythroblasts. In addition, a small population of the cells showed high expression levels of myeloid markers, Mac-1 and Gr-1 (Fig. 1D).

\section{Differentiation from GATA-1-null proerythroblasts to myeloid and mast cells}

High PU.1 expression and the small population of cells expressing myeloid markers imply that GATA-1-null proerythroblasts could retain the differentiation ability to myeloid lineages, and a small proportion of the cells could spontaneously differentiate to myeloid cells. To examine this possibility, we first analyzed the expression of various cytokine receptors in the unsorted cells and the FACS-purified CD71 ${ }^{\text {high }} \mathrm{Mac}-1^{-}$GATA-1-null cells by RT-PCR (Fig. 2A). mRNA of not only EPO receptor, but also myeloid lineage receptors GM-CSF receptor- $\alpha$ and $I L-3$ receptor- $\alpha$, were expressed in the GATA-1-null erythroid, namely CD71 ${ }^{\text {high }} \mathrm{Mac}-1^{-}$cells. The expression of $c$-fms, G-CSF receptor, and $I L-7$ receptor- $\alpha$ were detected in the unsorted GATA-1-null cells but not in the sorted GATA-1-null CD71 ${ }^{\text {high }}$ Mac- $1^{-}$ cells. These results showed that $c$-fms, G-CSF receptor, and $I L-7$ receptor- $\alpha$ were expressed in the GATA-1-null non-erythroid cells.

Responsiveness of the cells to the ligands of these receptors was analyzed (Fig. 2B). The cells responded to GM-CSF and IL-3 up until 1 wk after changing the culture from an EPO-containing medium to a GM-CSF- or IL-3-containing medium. Notably, the growth curves after changing media were similar to that of the EPO-containing medium. The GATA-1-null cells did not respond to M-CSF, G-CSF, or IL-7, which was consistent with the mRNA expression of the receptors of these cytokines (Fig. 2A). The cells cultured in the presence of GM-CSF differentiated to neutrophils and macrophages in a week, which was confirmed by morphological, phagocytic activity, and surface marker analyses (Fig. 2C,D). In contrast, IL-3 stimulation induced the differentiation to mast cells (Fig. 2E).

GATA-1-null proerythroblasts did not express c-mpl, thrombopoietin (TPO) receptor, and did not respond to TPO (Fig. 2A,B). However, erythroid and megakaryocytic cells have been reported to have closely related lineages (Debili et al. 1996; Era et al. 1997; Akashi et al. 2000; Vannucchi et al. 2000). Then, we examined whether reconstituted $\mathrm{TPO} / \mathrm{c}-\mathrm{mpl}$ signaling induced the differentiation to megakaryocytes after retroviral expression of 
A

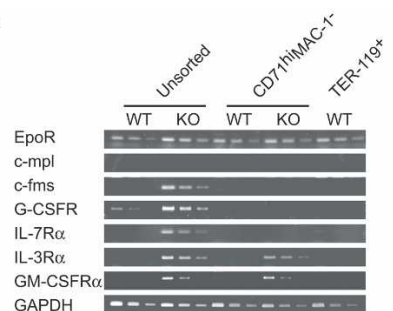

B

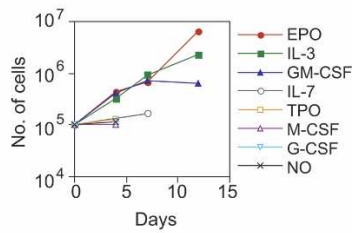

C



E
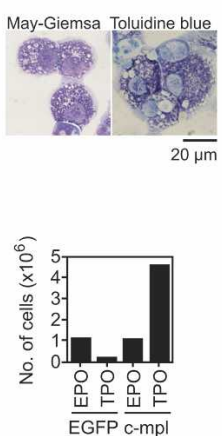

G

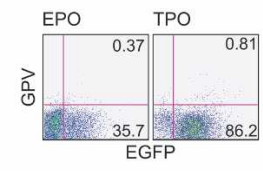

Figure 2. Responsiveness of GATA-1-null proerythroblasts to various cytokines. (A) RT-PCR analysis of cytokine receptors. (B) Number of cells after altering the condition from an EPO-containing medium to the media containing cytokines as described. Data are shown as mean \pm standard deviation $(n=6)$. (C) May-Giemsa staining and latex phagocytosis analysis of cells after culturing in the presence of GM-CSF for $7 \mathrm{~d}$. (D) FACS analysis of cells after culturing in the presence of GM-CSF for $7 \mathrm{~d}$. (E) May-Giemsa and toluidine blue staining of cells after culturing in the presence of IL-3 for $7 \mathrm{~d}$. $(F)$ Number of cells infected with control EGFP and $c$-mpl retrovirus $1 \mathrm{wk}$ after culturing in the presence of EPO or TPO as described. $(G)$ Expression of a megakaryocyte marker GPV on the c-mpl-expressing cells 1 wk after culturing in the presence of EPO or TPO as described.

$c$-mpl. Although expression of c-mpl induced TPO-dependent cell proliferation, no megakaryocytic differentiation took place (Fig. 2F,G). These data suggest that the GATA-1-null proerythroblasts have lost the differentiation capacity to megakaryocytic lineage. To analyze the in vivo differentiation activity to lymphoid lineage cells, transplantation of the GATA-1-null proerythroblasts to sublethally irradiated NOD/SCID mice was carried out. GATA-1-null proerythroblasts did not contribute to lymphoid, erythroid, or myeloid repopulation in the mice 4 wk after the transplantation (data not shown). Considering that the survival of the GATA-1-null cells required the cytokine stimulation of EPO, GM-CSF, or IL-3 (Fig. 2B), the defective myeloid differentiation in vivo would probably be due to some inappropriate microenvironment in the transplanted mice.

\section{Differentiation dynamics to myeloid cells}

When the cells were stained with anti-Mac-1 and antiCD71 antibodies, the vast majority of GATA-1-null cells belonged to the $\mathrm{CD} 71^{\text {high }} \mathrm{Mac}-1^{-}$population. A significant percentage of the cells other than this major population expressed Mac-1. There are two possibilities to explain the existence of these two populations. One is that both $\mathrm{CD} 71^{\text {high }} \mathrm{Mac}-1^{-}$cells and $\mathrm{Mac}-\mathrm{1}^{+}$cells proliferate simultaneously, and the other is that one population is the progeny of the other. When sorted $\mathrm{CD} 71^{\text {high }} \mathrm{Mac}-1^{-}$cells were cultured in the presence of EPO, the cells gave rise to both populations (Fig. 3A). In contrast, CD $71^{\text {low/- }} \mathrm{Mac}^{-1^{+}}$cells did not produce CD71 ${ }^{\text {high }} \mathrm{Mac}^{-} 1^{-}$cells (data not shown).

Growth of the cells after altering the cytokines from EPO to GM-CSF was quite similar to the growth in the presence of EPO (Fig. 2B), suggesting that the majority of the cells responded to GM-CSF. This indicates that $\mathrm{CD} 71^{\text {high }}$ Mac-1 ${ }^{-}$cells responded to GM-CSF. It is unlikely that only the minor population that did not belong to the $\mathrm{CD} 71^{\text {high }} \mathrm{Mac}-1^{-}$population responded to GMCSF, because the minor population was $<10 \%$ and the kinetics of proliferation after changing the cytokines was very fast. To confirm that the small fraction of $\mathrm{CD} 71^{\text {low } /-} \mathrm{Mac}-1^{+}$cells was not propagated preferentially in the GM-CSF culture, the sorted CD $71^{\text {high }} \mathrm{Mac}^{-} 1^{-}$cells were cultured in the presence of GM-CSF. As shown in Figure $3 \mathrm{~B}$, the $\mathrm{CD} 71^{\text {high }} \mathrm{Mac}-1^{-}$cells decreased gradually, and the CD $71^{\text {low } /-}$ Mac- $1^{+}$cells reciprocally increased after switching the cytokines. The sorted CD $71^{\text {high }} \mathrm{Mac}^{-}{ }^{-}$ cells exhibited the characteristics of macrophage and neutrophil after culturing in the presence of GM-CSF (data not shown). Next, we carried out cloning experiments using a limiting dilution method. CD7 $11^{\text {high }} \mathrm{Mac}^{-} 1^{-}$ cells were sorted, and cloned cells were propagated in the presence of EPO. The cells were then transferred to the culture containing GM-CSF. The data of two representative clones are shown in Figure 3, C and D. Again, EPO-responsive CD $71^{\text {high }}$ Mac- $1^{-}$clonal cells gave rise to $\mathrm{CD} 71^{\text {low/ }}-\mathrm{Mac}-1^{+}$cells in the presence of EPO, and differentiated to neutrophils and macrophages in the presence of GM-CSF.

A
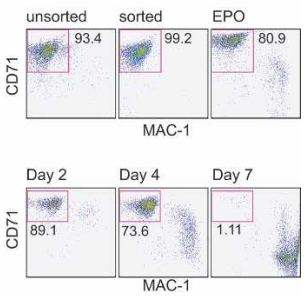

C

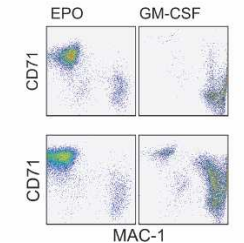

Figure 3. Differentiation from $\mathrm{CD} 71^{\text {high }} \mathrm{Mac}-1^{-}$cells to myeloid cells. (A) Expression of CD71 and Mac-1 on unsorted cells, sorted cells, and the cells cultured for $1 \mathrm{wk}$ in the presence of EPO after sorting. (B) Transition from the sorted CD71 ${ }^{\text {high }} \mathrm{Mac}-1^{-}$cells to myeloid cells in the presence of GM-CSF. (C) Expression of CD71 and Mac-1 on the cloned GATA-1-null proerythroblasts in the presence of EPO or GM-CSF. 
A

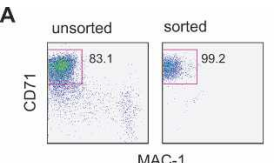

B

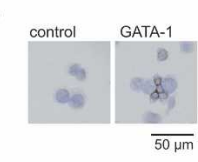

C
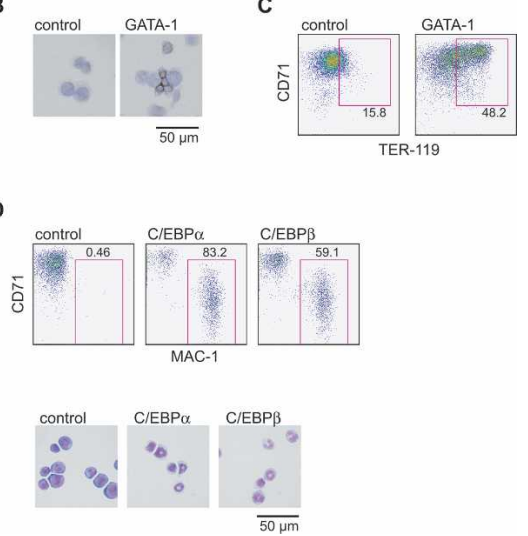

Figure 4. Terminal differentiation induced by retroviral gene expression. (A) Expression of CD71 and Mac-1 on the unsorted cells and the sorted cells. (B) Terminal erythroid differentiation using GATA-1-expressing retrovirus. Benzidine staining is shown. $(C)$ CD71 and TER-119 expression after the infection of GATA-1 retrovirus. $(D)$ Expression of CD71 and Mac-1 after retroviral introduction of $\mathrm{C} / \mathrm{EBP} \alpha$ and $\mathrm{C} / \mathrm{EBP} \beta$. (E) May-Giemsa staining of the cells infected with control, $\mathrm{C} / \mathrm{EBP} \alpha$, and $\mathrm{C} / \mathrm{EBP} \beta$ retrovirus and cultured for $1 \mathrm{wk}$ in the presence of EPO.

\section{Terminal differentiation by forced expression} of GATA-1 and C/EBP

We analyzed the effect of transcription factors on cell differentiation using retroviral gene transduction. The first experiment is the recapitulation of GATA-1 expression in the GATA-1-null proerythroblasts. The CD $71^{\text {high }}$ Mac- $^{-}$cells were purified (Fig. 4A) and infected with the retroviruses. Two days after the infection of retrovirus expressing GATA-1 and EYFP, EYFP-positive cells were sorted and analyzed. Benzidine-positive cells appeared, and more than half of the infected cells exhibited a high expression of Ter-119 (Fig. 4B,C). In addition, enucleated erythrocytes appeared $4 \mathrm{~d}$ after the infection (data not shown). These data show that only a relatively short period was necessary to complete the terminal erythroid differentiation of GATA-1-null proerythroblasts. When the retroviral-infected cells were cultured in the presence of TPO, none of the megakaryocyte markers such as GPIIb, GPV, and acetylcholine esterase was expressed (data not shown), suggesting that megakaryocytic differentiation was not recapitulated by the expression of GATA-1.

$\mathrm{C} / \mathrm{EBP} \alpha$ or $\mathrm{C} / \mathrm{EBP} \beta$ are crucial transcription factors in myeloid differentiation, and overexpression of the factors can induce myeloid differentiation (Heavey et al. 2003; Xie et al. 2004). Retroviral overexpression of C/EBP $\alpha$ and C/EBP $\beta$ induced the differentiation of the GATA-1-null CD71 ${ }^{\text {high }}$ Mac-1 $^{-}$proerythroblasts to $\mathrm{CD} 71^{\text {low/- }}$ Mac- $1^{+}$neutrophils in the presence of EPO (Fig. 4D). These data show that EPO signaling is not inhibitory to granulocytic differentiation under the GATA-1-null condition. Although the GATA-1-null CD71 ${ }^{\text {high }} \mathrm{Mac}^{-1^{-}}$cells expressed GATA-2, Fog-1, and Scl
(Fig. 1F), all of which were implicated in the regulation of erythropoiesis, expression of those factors was not sufficient for the definite commitment to the erythroid lineage.

\section{Differentiation status and potential of GATA-1-null proerythroblasts}

It is difficult to precisely determine to which differentiation stage the GATA-1-null erythroid cells belong, because the GATA-1-null erythroid cells could have resulted from some aberrant differentiation. However, it is conceivable that the cells are similar to proerythroblasts for the following reasons: (1) the morphology and erythroid marker expression, and (2) the short period for the completion of the terminal differentiation. Completion of erythroid differentiation in 2-4 d after the recapitulation of GATA-1 verifies that the GATA-1-null cells are the committed, relatively mature erythroid cells (Fig. 4B).

Characteristics of the GATA-1-null proerythroblastic cells are summarized in Figure 5. The erythroid-committed cells possess the differentiation ability to become macrophages, neutrophils, and mast cells, but cannot differentiate to lymphoid cells or megakaryocytes. It is possible that GATA-1-null proerythroblasts could not give rise to lymphoid cells, since erythroid and lymphoid lineages are distantly separated from the standpoint of hematopoietic lineages. It is intriguing that megakaryocytic differentiation cannot be achieved even when $\mathrm{TPO} / c-m p l$ signaling was restored in the GATA-1-null erythroid cells. Even though erythroid and megakaryocytic lineages are considered quite close and share various molecular mechanisms in differentiation, there may exist some specific molecular mechanisms prohibiting differentiation to an adjacent cell lineage. Recently, Weiss and colleagues (Stachura et al. 2006) found that common megakaryocyte-erythroid precursor (MEP) cells could be obtained by the differentiation of GATA-null ES cells with the OP9 system. They added TPO instead of EPO during the differentiation induction and obtained MEP cells. In both cases, additional mutation(s) does not seem to be necessary. It remains to be elucidated how the different cytokine signals induce different multipotent progenitor cells with different phenotypes.

In zebrafish, hematopoietic differentiation alteration under the GATA-1-null condition was analyzed during ontogeny (Galloway et al. 2005). Loss of GATA-1 transforms primitive blood precursors into myeloid cells in vivo and brought about subsequent massive expansion of granulocytic neutrophils and macrophages at the expense of red blood cells. Although the study did not define whether the conversion to myeloid lineage cells oc-

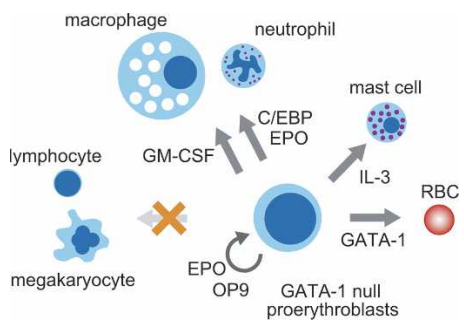

Figure 5. Diagram of the differentiation activity of GATA-1-null proerythroblasts. 
curred via committed erythroid cells, our present data of multipotential proerythroblasts is consistent with the phenotype of zebrafish GATA-1-null hematopoiesis. Thus, in both fish and mammals, the absence of erythroid terminal differentiation brought about differentiation capacity to the myeloid lineages.

\section{Lineage instability of the cells lacking the transcription factor essential for differentiation}

B-cell commitment and development requires the stepwise activation of multiple transcription factors, including the basic helix-loop-helix (bHLH) E2A proteins and the paired homeodomain-containing protein Pax5 (Urbánek et al. 1994; Zhuang et al. 1994). Pro-B cells lacking Pax 5 or E2A have been reported to function as pluripotent hematopoietic progenitors (Mikkola et al. 2002; Ikawa et al. 2004). These pro-B cells exhibit promiscuous gene expression, such as myeloid and erythroid lineages, and possess the capability to differentiate to T, NK, and myeloid lineages. Since wild-type pro-B cells can be propagated with the stimulation of IL-7 and SCF without further differentiation, it is conceivable that Pax5- and E2A-null pro-B cells could be maintained similarly.

Differentiation-blocked pro-B cells lacking the transcription factors essential for differentiation could have been exceptional cases. Our present study, however, indicates that such lineage infidelity would be a more general rule when the transcription factors essential for subsequent differentiation are nullified. The other important point is that not only very immature and potentially propagative cells such as pro-B cells but also the committed cells still possess the ability to differentiate to other lineages.

Compared with B lymphoid cells, it is extremely difficult to culture myeloid and erythroid lineages maintaining a specific differentiation stage. It is surprising that relatively mature erythroid-committed proerythroblasts gained long-term proliferation ability when the transcription factor necessary for terminal differentiation was eliminated. Notably, GATA-1-null proerythroblasts differentiated to granulocytes, macrophages, and mast cells, but not to megakaryocytes or lymphoid cells. It is conceivable that GATA-1-null proerythroblasts are relatively mature, compared with Pax5- and E2A-null pro-B cells, from the view of the differentiation process. Therefore, the relatively limited differentiation ability of the GATA-1-null erythroblasts could be attributed to the maturation level of the cells. Meanwhile, the GATA-1null cells could not differentiate to megakaryocytes in spite of the close lineage relationship between erythroid and megakaryocytic lineages. There could be some active mechanisms to inhibit the differentiation to the closely related lineage.

Recently, it has been reported that the fate of committed hematopoietic progenitors can be altered by cytokine siginaling or enforced expression of a transcription factor (Kondo et al. 2000; Iwasaki et al. 2003; Iwasaki-Arai et al. 2003, Xie et al. 2004). Moreover, the cells that once expressed a lineage marker possess the ability to differentiate into the other lineages. Our data strongly support the novel notion of the differentiation flexibility of the committed cells. Namely, although commitment proceeds gradually and the differentiation ability is reduced along the differentiation progression, even the commit- ted cells possess the differentiation ability into lineages other than the committed lineage.

\section{Materials and methods}

Cells

Cultures of wild-type E14tg2a (WT) and GATA-1-null (KO) ES cells (Zheng et al. 2006) were maintained as previously described (Kitajima et al. 2002). The hematopoietic differentiation induction of ES cells on OP9 stromal cells has been described (Nakano et al. 1994, 1996). Human EPO $(2 \mathrm{U} / \mathrm{mL})$, murine GM-CSF $(10 \mathrm{ng} / \mathrm{mL})$, murine IL-3, murine IL-7 (10 $\mathrm{ng} / \mathrm{mL})$, human TPO $(10 \mathrm{ng} / \mathrm{mL})$, human M-CSF $(10 \mathrm{ng} / \mathrm{mL})$, and G-CSF $(10 \mathrm{ng} / \mathrm{mL})$ were added in the culture as indicated. Phagocytosis was examined by incubation of cells with the microsphere (Fluoresbrite carboxylate $0.75-\mu \mathrm{m}$ microspheres, Polysciences, Inc.).

Antibodies, staining, and analysis

Biotinylated TER-119 antibody was a kind gift from Dr T. Kina (Kyoto University, Kyoto, Japan) (Ikuta et al. 1990). PE-conjugated anti-CD71 (e-Bioscience), biotinylated anti-GPV (Seikagaku Corp.) (Takada et al. 1995), anti-GPIIb, biotinylated Mac-1, biotinylated or PE-conjugated GR1, FITC-conjugated FCR $\gamma$, biotinylated B220, biotinylated Thy-1.2, biotinylated or APC/Cy7-conjugated anti-c-KIT, PE-conjugated anti-CD34, and biotinylated or APC-conjugated SCA-1 antibodies (Pharmingen) were used for FACS analysis. The biotinylated antibodies were visualized by PE- or Cychrome-conjugated streptavidin (Pharmingen), and the GPIIb antibody was visualized by PE-conjugated anti-Rat IgG antibody (Pharmingen). The cells were stained with the antibodies as previously described (Kitajima et al. 2002). The analysis of stained cells and cell sorting were done by FACSCalibur and FACSAria (Becton Dickinson). Cell cycle was analyzed by staining cells with propidium iodide.

RT-PCR analysis

Total RNA was isolated using RNeasy (Qiagen) and reverse transcribed by the Thermoscript RT-PCR system (Invitrogen). The cDNA was diluted in series, and PCR was performed for 25-30 cycles using specific primers whose sequences will be provided on request.

Retrovirus transduction

Retrovirus plasmid vector pMY.IRES.EGFP, human c-mpl cDNA, and PLAT-E ecotropic packaging cell line (Morita et al. 2000) were kind gifts from Dr. T. Kitamura (Tokyo University, Tokyo, Japan). pMY.IRES.EYFP was constructed by replacing the EGFP gene of pMY.IRES.EGFP to EYFP gene purchased from Clontech. GATA-1, C/EBP $\alpha$, and C/EBP $\beta$ cDNAs were inserted into the multicloning site (MCS) of pMY.IRES.EYFP, and c-mpl cDNA was inserted into the MCS of pMY.IRES.EGFP. The retrovirus supernatant was obtained by a transient transfection of the retrovirus plasmid vectors to the PLAT-E packaging cell line using Lipofectamine 2000 (Invitrogen). The infection was done by spinoculation $(2 \mathrm{~h}$, $25^{\circ} \mathrm{C}, 2500 \mathrm{rpm}$ ). Retroviruses expressing GATA-1, C/EBP $\alpha$, and C/EBP $\beta$ were infected to the GATA-1-null CD71 ${ }^{\text {high }} \mathrm{MAC}-1^{-}$erythroid cells.

\section{Acknowledgments}

We thank Drs. Yamamoto and Minegishi for the kind gift of GATA-1 genomic DNA and cDNA; Dr. Kitamura for Plat-E cells, c-mpl cDNA, and pMY.IRES.EGFP; and Dr. Iwama for cDNAs of C/EBP $\alpha$ and $\mathrm{C} / \mathrm{EBP} \beta$. This work was supported in part by grants from the MEXT, NEDO, the Uehara Memorial Foundation, the Osaka Cancer Foundation, and the 21st Century COE "CICET."

\section{References}

Akashi, K., Traver, D., Miyamoto, T., and Weissman, I.L. 2000. A clonogenic common myeloid progenitor that gives rise to all myeloid lineages. Nature 404: 193-197.

Cantor, A.B. and Orkin, S.H. 2001. Hematopoietic development: A balancing act. Curr. Opin. Genet. Dev. 11: 513-519.

Debili, N., Coulombel, L., Croisille, L., Katz, A., Guichard, J., BretonGorius, J., and Vainchenker, W. 1996. Characterization of a bipotent erythro-megakaryocytic progenitor in human bone marrow. Blood 
88: $1284-1296$.

DeKoter, R.P. and Singh, H. 2000. Regulation of B lymphocyte and macrophage development by graded expression of PU.1. Science 288: 1439-1441.

Era, T., Takahashi, T., Sakai, K., Kawamura, K., and Nakano, T. 1997. Thrombopoietin enhances proliferation and differentiation of murine yolk sac erythroid progenitors. Blood 89: 1207-1213.

Ferreira, R., Ohneda, K., Yamamoto, M., and Philipsen, S. 2005. GATA1 function, a paradigm for transcription factors in hematopoiesis. Mol. Cell. Biol. 25: 1215-1227.

Fujiwara, Y., Browne, C.P., Cunniff, K., Goff, S.C., and Orkin, S.H. 1996 Arrested development of embryonic red cell precursors in mouse embryos lacking transcription factor GATA-1. Proc. Nat. Acad. Sci. 93: 12355-12358.

Galloway, J.L., Wingert, R.A., Thisse, C., Thisse, B., and Zon, L.I. 2005. Loss of gatal but not gata 2 converts erythropoiesis to myelopoiesis in zebrafish embryos. Dev. Cell 8: 109-116.

Heavey, B., Charalambous, C., Cobaleda, C., and Busslinger, M. 2003. Myeloid lineage switch of Pax5 mutant but not wild-type B cell progenitors by $\mathrm{C} / \mathrm{EBP} \alpha$ and GATA factors. EMBO I. 22: 3887-3897.

Ikawa, T., Kawamoto, H., Wright, L.Y., and Murre, C. 2004. Long-term cultured E2A-deficient hematopoietic progenitor cells are pluripotent. Immunity 20: 349-360.

Ikuta, K., Kina, T., MacNeil, I., Uchida, N., Peault, B., Chien, Y.H., and Weissman, I.L. 1990. A developmental switch in thymic lymphocyte maturation potential occurs at the level of hematopoietic stem cells. Cell 62: 863-874.

Iwasaki, H., Mizuno, S., Wells, R.A., Cantor, A.B., Watanabe, S., and Akashi, K. 2003. GATA-1 converts lymphoid and myelomonocytic progenitors into the megakaryocyte/erythrocyte lineages. Immunity 19: 451-462.

Iwasaki-Arai, J., Iwasaki, H., Miyamoto, T., Watanabe, S., and Akashi, K. 2003. Enforced granulocyte/macrophage colony-stimulating factor signals do not support lymphopoiesis, but instruct lymphoid to myelomonocytic lineage conversion. J. Exp. Med. 197: 1311-1322.

Kimura, T., Sonoda, Y., Iwai, N., Satoh, M., Yamaguchi-Tsukio, M., Izui, T., Suda, M., Sasaki, K., and Nakano, T. 2000. Proliferation and cell death of embryonic primitive erythrocytes. Exp. Hematol. 28: 635641.

Kitajima, K., Masuhara, M., Era, T., Enver, T., and Nakano, T. 2002. GATA-2 and GATA-2/ER display opposing activities in the development and differentiation of blood progenitors. EMBO I. 21: 30603069.

Kondo, M., Scherer, D.C., Miyamoto, T., King, A.G., Akashi, K., Sugamura, K., and Weissman, I.L. 2000. Cell-fate conversion of lymphoid-committed progenitors by instructive actions of cytokines. $\mathrm{Na}$ ture 407: 383-386.

Kulessa, H., Frampton, J., and Graf, T. 1995. GATA-1 reprograms avian myelomonocytic cell lines into eosinophils, thromboblasts, and erythroblasts. Genes \& Dev. 9: 1250-1262.

Mikkola, I., Heavey, B., Horcher, M., and Busslinger, M. 2002. Reversion of B cell commitment upon loss of Pax5 expression. Science 297: $110-113$

Morceau, F., Schnekenburger, M., Dicato, M., and Diederich, M. 2004. GATA-1: Friends, brothers, and coworkers. Ann. N.Y. Acad. Sci. 1030: $537-554$

Morita, S., Kojima, T., and Kitamura, T. 2000. Plat-E: An efficient and stable system for transient packaging of retroviruses. Gene Ther. 7: 1063-1066.

Nakano, T., Kodama, H., and Honjo, T. 1994. Generation of lymphohematopoietic cells from embryonic stem cells in culture. Science 265: 1098-1101.

- 1996. In vitro development of primitive and definitive erythrocytes from different precursors. Science 272: 722-724.

Pan, X., Ohneda, O., Ohneda, K., Lindeboom, F., Iwata, F., Shimizu, R., Nagano, M., Suwabe, N., Philipsen, S., Lim, K.C., et al. 2005. Graded levels of GATA-1 expression modulate survival, proliferation and differentiation of erythroid progenitors. J. Biol. Chem. 280: 2238522394.

Pevny, L., Simon, M.C., Robertson, E., Klein, W.H., Tsai, S.F., D’Agati, V., Orkin, S.H., and Costantini, F. 1991. Erythroid differentiation in chimaeric mice blocked by a targeted mutation in the gene for transcription factor GATA-1. Nature 349: 257-260.
Pevny, L., Lin, C.S., D'Agati, V., Simon, M.C., Orkin, S.H., and Costantini, F. 1995. Development of hematopoietic cells lacking transcription factor GATA-1. Development 121: 163-172.

Scott, E.W., Simon, M.C., Anastasi, J., and Singh, H. 1994. Requirement of transcription factor PU.1 in the development of multiple hematopoietic lineages. Science 265: 1573-1577.

Stachura, D.L., Chou, S.T., and Weiss, M.J. 2006. An early block to erythro-megakaryocytic development conferred by loss of transcription factor GATA-1. Blood 107: 87-97.

Takada, K., Saito, M., Kaneko, H., Iizuka, K., Kokai, Y., and Fujimoto, J. 1995. Novel monoclonal antibody reactive with thrombin-sensitive 74-kDa glycoproteins present on platelets and megakaryocytes both from mouse and rat. Hybridoma 14: 361-367.

Tanaka, M., Zheng, J., Kitajima, K., Kita, K., Yoshikawa, H., and Nakano, T. 2004. Differentiation status dependent function of FOG-1. Genes Cells 9: 1213-1226.

Urbánek, P., Wang, Z.Q., Fetka, I., Wagner, E.F., and Busslinger, M. 1994 Complete block of early B cell differentiation and altered patterning of the posterior midbrain in mice lacking Pax5/BSAP. Cell 79: 901912.

Vannucchi, A.M., Paoletti, F., Linari, S., Cellai, C., Caporale, R., Ferrini, P.R., Sanchez, M., Migliaccio, G., and Migliaccio, A.R. 2000. Identification and characterization of a bipotent (erythroid and megakaryocytic) cell precursor from the spleen of phenylhydrazine-treated mice. Blood 95: 2559-2568.

Walsh, J.C., DeKoter, R.P., Lee, H.J., Smith, E.D., Lancki, D.W., Gurish, M.F., Friend, D.S., Stevens, R.L., Anastasi, J., and Singh, H. 2002. Cooperative and antagonistic interplay between PU.1 and GATA-2 in the specification of myeloid cell fates. Immunity 17: 665-676.

Weiss, M.J. and Orkin, S.H. 1995. Transcription factor GATA-1 permits survival and maturation of erythroid precursors by preventing apoptosis. Proc. Nat. Acad. Sci. 92: 9623-9627.

Weiss, M.J., Keller, G., and Orkin, S.H. 1994. Novel insights into erythroid development revealed through in vitro differentiation of GATA-1 embryonic stem cells. Genes \& Dev. 8: 1184-1197.

Welch, J.J., Watts, J.A., Vakoc, C.R., Yao, Y., Wang, H., Hardison, R.C. Blobel, G.A., Chodosh, L.A., and Weiss, M.J. 2004. Global regulation of erythroid gene expression by transcription factor GATA-1. Blood 104: $3136-3147$.

Xie, H., Ye, M., Feng, R., and Graf, T. 2004. Stepwise reprogramming of B cells into macrophages. Cell 117: 663-676.

Zheng, J., Kitajima, K., Sakai, E., Kimura, T., Minegishi, N., Yamamoto, M., and Nakano, T. 2006. Differential effects of GATA-1 on proliferation and differentiation of erythroid lineage cells. Blood 107: 520 527.

Zhuang, Y., Soriano, P., and Weintraub, H. 1994. The helix-loop-helix gene E2A is required for B cell formation. Cell 79: 875-884. 


\section{Erratum}

Genes \& Development 20: 654-659 (2006)

Multipotential differentiation ability of GATA-1-null erythroid-committed cells

Kenji Kitajima, Jie Zheng, Hilo Yen, Daijiro Sugiyama, and Toru Nakano

Due to an error, the received and accepted dates published for the above-mentioned paper were incorrect. The correct dates are as follows:

Received September 22, 2005; revised version accepted January 18, 2006.

We apologize for the error. 


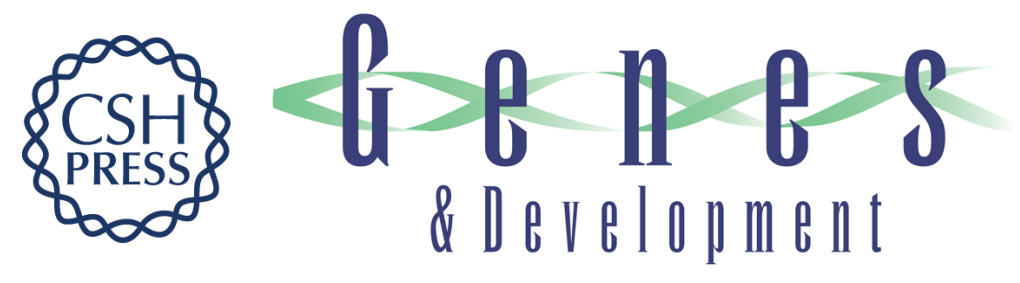

\section{Multipotential differentiation ability of GATA-1-null erythroid-committed cells}

Kenji Kitajima, Jie Zheng, Hilo Yen, et al.

Genes Dev. 2006, 20:

Access the most recent version at doi:10.1101/gad.1378206

\section{Related Content}

\section{Erratum}

Genes Dev. UNKNOWN , 2006 20: 1974
References This article cites 38 articles, 21 of which can be accessed free at: http://genesdev.cshlp.org/content/20/6/654.full.htmI\#ref-list-1

Articles cited in:

http://genesdev.cshlp.org/content/20/6/654.full.htmI\#related-urls

\section{License}

Email Alerting Service
Receive free email alerts when new articles cite this article - sign up in the box at the top right corner of the article or click here.

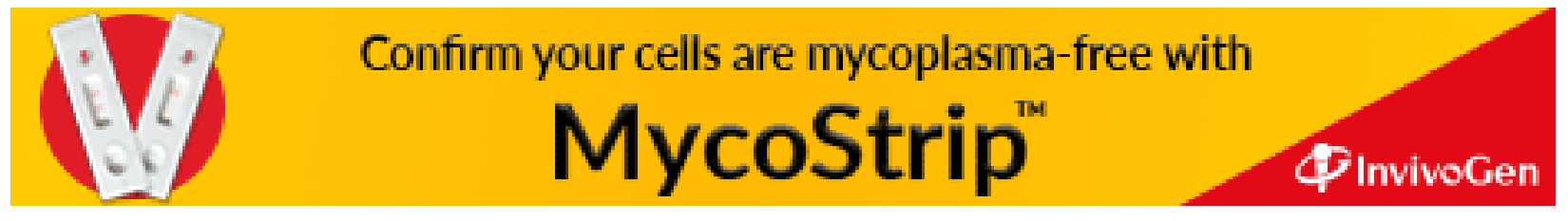

